\title{
Protective Effect of Carvacrol against Gut Dysbiosis and Clostridium difficile Associated Disease in a Mouse Model
}

\author{
Shankumar Mooyottu ${ }^{1}$, Genevieve Flock ${ }^{1}$, Abhinav Upadhyay ${ }^{1}$, Indu Upadhyaya ${ }^{1}$, \\ Kendra Maas $^{2}$ and Kumar Venkitanarayanan ${ }^{1 *}$ \\ ${ }^{1}$ Department of Animal Science, University of Connecticut, Storrs, CT, USA, ${ }^{2}$ Microbial Analysis, Resources, and Services, \\ University of Connecticut, Storrs, CT, USA
}

OPEN ACCESS

Edited by:

Tzi Bun Ng,

The Chinese University of Hong Kong,

Hong Kong

Reviewed by:

Xingmin Sun,

University of South Florida, USA

Atte Von Wright

University of Eastern Finland, Finland

${ }^{*}$ Correspondence:

Kumar Venkitanarayanan

kumar.venkitanarayanan@uconn.edu

Specialty section:

This article was submitted to Antimicrobials, Resistance and

Chemotherapy,

a section of the journal

Frontiers in Microbiology

Received: 22 January 2017 Accepted: 28 March 2017

Published: 21 April 2017

Citation:

Mooyottu S, Flock G, Upadhyay A,

Upadhyaya I, Maas K and

Venkitanarayanan K (2017) Protective

Effect of Carvacrol against Gut

Dysbiosis and Clostridium difficile

Associated Disease in a Mouse

Model. Front. Microbiol. 8:625.

doi: 10.3389/fmicb.2017.00625
This study investigated the effect of carvacrol (CR), a phytophenolic compound on antibiotic-associated gut dysbiosis and $C$. difficile infection in a mouse model. Five to sixweek-old C57BL/6 mice were randomly divided into seven treatment groups (challenge and control) of eight mice each. Mice were fed with irradiated feed supplemented with CR (0, 0.05, and 0.1\%); the challenge groups were made susceptible to $C$. difficile by orally administering an antibiotic cocktail in water and an intra-peritoneal injection of clindamycin. Both challenge and control groups were infected with $10^{5} \mathrm{CFU} / \mathrm{ml}$ of hypervirulent C. difficile (ATCC 1870) spores or PBS, and observed for clinical signs for 10 days. Respective control groups for CR, antibiotics, and their combination were included for investigating their effect on mouse enteric microflora. Mouse body weight and clinical and diarrhea scores were recorded daily post infection. Fecal samples were collected for microbiome analysis using rRNA sequencing in MiSeq platform. Carvacrol supplementation significantly reduced the incidence of diarrhea and improved the clinical and diarrhea scores in mice $(p<0.05)$. Microbiome analysis revealed a significant increase in Proteobacteria and reduction in the abundance of protective bacterial flora in antibiotic-treated and $C$. difficile-infected mice compared to controls $(p<0.05)$. However, CR supplementation positively altered the microbiome composition, as revealed by an increased abundance of beneficial bacteria, including Firmicutes, and significantly reduced the proportion of detrimental flora such as Proteobacteria, without significantly affecting the gut microbiome diversity compared to control. Results suggest that CR could potentially be used to control gut dysbiosis and reduce $C$. difficile infection.

Keywords: Clostridium difficile, carvacrol, microbiome, gut dysbiosis, mouse model

\section{INTRODUCTION}

Clostridium difficile infection is the major cause of antibiotic-associated diarrhea in hospital settings around the world (McFarland, 2008; Hookman and Barkin, 2009). C. difficile principally causes a serious toxin-mediated colitis in the elderly and immunocompromised patients (Weese, 2010). Annually, more than 300,000 cases of $C$. difficile associated diseases (CDAD) are reported in the United States, resulting in more than US\$3 billion as health care costs (Wilkins and Lyerly, 2003; Ghose et al., 2007). A recently emerged, highly toxigenic and hyper-virulent $C$. difficile strain 
NAP1/ribotype 027 has been implicated in increasing incidence of CDAD among patients all over the world (Sunenshine and McDonald, 2006; Blossom and McDonald, 2007; Hookman and Barkin, 2009).

C. difficile infection has been associated with the use of antibiotics and gastric acid suppressing agents that result in gut dysbiosis (Bartlett, 1992; Kelly and LaMont, 1998; Dial et al., 2005). Prolonged antibiotic therapy results in the disruption of the normal enteric microflora, leading to an altered microbial composition such as increased population of Proteobacteria and reduced proportion of Bacteroides and Firmicutes in the gut microbiome (Shahinas et al., 2012; Ling et al., 2014; Seekatz and Young, 2014; Theriot et al., 2014). Consequently, gut dysbiosis results in the germination of spores and selection for $C$. difficile in the intestine. Following spore germination and outgrowth in the presence of a disrupted gut flora, the vegetative cells of $C$. difficile produce potent toxins known as toxin A and toxin B (Voth and Ballard, 2005). Clostridium difficile toxins (A and $\mathrm{B}$ ) are functionally glucosyl transferases, which inactivate the Rho family GTPases associated with Factin regulation, and cause disruption of the cytoskeleton and intestinal epithelial tight junctions (von Eichel-Streiber et al., 1999; Keel and Songer, 2006). This leads to a severe inflammatory response with the release of cytokines and leukotrienes, causing pseudomembrane formation and severe diarrhea (McDonald et al., 2006; Sunenshine and McDonald, 2006; Hookman and Barkin, 2009). Since gut dysbiosis is considered as the most important predisposing factor in CDAD, emerging and novel therapeutic approaches, including fecal microbiome transplantation (FMT) primarily aimed at restoration of the normal gut flora in CDAD patients are explored (Kassam et al., 2013).

Despite the fact that a majority of the currently used antibiotics can predispose CDAD by disrupting the normal gut flora, antibiotics are still used as the primary line of treatment against infection (Bartlett, 1992; O'Connor et al., 2004). Nonetheless, many of the anti- C. difficile antibiotics are found to predispose $\mathrm{CDAD}$ in patients by inducing gut dysbiosis (O'Connor et al., 2004; McFarland, 2008; Shah et al., 2010). Moreover, the emergence of antibiotic resistant strains of hypervirulent $C$. difficile is documented worldwide (Spigaglia et al., 2011; Steiner et al., 2012). The Centers for Disease Control and Prevention (CDC) recently listed C. difficile as one among the three urgent threats in their report on emerging pathogens with antibiotic resistance (Steiner et al., 2012). Since the toxins are the major virulence factors for CDAD, a search for alternative therapeutic agents, which can reduce $C$. difficile virulence without affecting normal gastrointestinal flora opens a new research area.

Carvacrol (CR) is a food grade, monoterpenoid phytophenol that is naturally present in oregano and thyme oil. Diverse pharmacological actions of carvacrol, including antimicrobial and anti-inflammatory activities have been previously demonstrated (Baser, 2008). A recent study from our laboratory suggested the potential use of $\mathrm{CR}$ as an anti-C. difficile therapeutic agent due to its inhibitory effect on $C$. difficile toxin production without affecting the growth of beneficial gut bacteria in vitro (Mooyottu et al., 2014a). This study demonstrated that CR significantly inhibited toxin production in hypervirulent $C$. difficile strains by modulating toxin production genes. Therefore, this study investigated the therapeutic effect of CR against $C$. difficile in an in vivo model, specifically its impact on the clinical course of $C$. difficile infection and the host microbiome. Mouse is a well-established model of $C$. difficile infection (Chen et al., 2008; Sun et al., 2011), and antibiotic-associated C. difficile infection can be induced in a mouse model by administering antibiotics orally and intraperitoneally, followed by inoculation of C. difficile spores (Chen et al., 2008; Sun et al., 2011).

\section{MATERIALS AND METHODS}

\section{Ethics Statement, Animals, and Housing}

This study was conducted with the approval of the Institutional Animal Care and Use Committee (IACUC) of the University of Connecticut. All recommended guidelines for the care and use of animals were followed. Six-week-old C57BL/6 mice were obtained from Charles River (Boston, MA). Animals were housed in a biohazard level II, AALAC-accredited facility and monitored twice daily for health. Mice were provided with autoclaved food, water, and bedding, with 12-h light/dark cycles. All cage changes, $C$. difficile spore infection, and sample collections were performed under a laminar flow hood using properpersonal protective equipment. The work area was sterilized using 10\% bleach between experimental treatment groups to prevent crosscontamination. The mice were housed in pairs in a cage, and four cages were included for each treatment in each of the experiments.

\section{Mouse Model of C. difficile Infection and Treatment Groups}

The infection model adopted for this study is a modification of the method described by Chen et al. (2008). Five to six-weekold female mice were randomly divided into eight treatment groups of eight animals each (Table 1). The animals were subjected to food restriction for $12 \mathrm{~h}$, and given powdered feed supplemented with $0,0.05$, and $0.10 \%$ of CR. After 7 days, an antibiotic mixture was added in drinking water (kanamycin, $0.4 \mathrm{mg} / \mathrm{mL}$, gentamicin, $0.03 \mathrm{mg} / \mathrm{mL}$, colistin, 850 $\mathrm{U} / \mathrm{mL}$, metronidazole, $0.215 \mathrm{mg} / \mathrm{mL}$, and vancomycin, 0.045 $\mathrm{mg} / \mathrm{mL}$ ) for 3 days [challenge groups (Ant $+\mathrm{CD}$, Ant $+\mathrm{CD}+$ CR 0.05\%, and Ant $+\mathrm{CD}+\mathrm{CR} 0.1 \%$ ), and the antibiotic control group (Ant control)]. After the antibiotic treatment, the mice were given regular autoclaved water for 2 days, and all animals in the challenge groups (Ant $+\mathrm{CD}$, Ant $+\mathrm{CD}+\mathrm{CR} 0.05 \%$, and Ant $+\mathrm{CD}+\mathrm{CR} 0.1 \%$ ), and the antibiotic control group (Ant control) received a single dose of clindamycin $(10 \mathrm{mg} / \mathrm{kg}$, maximum volume of injection $0.5 \mathrm{ml} /$ mouse using a 27 gauge gavage needle and syringe) intraperitoneally 1 day before $C$ difficile challenge. This antibiotic pre-treatment was intended to disrupt the normal gut flora of mice and facilitate $C$. difficile colonization. All animals in the challenge groups (Ant $+\mathrm{CD}$, Ant $+\mathrm{CD}+\mathrm{CR} 0.05 \%$, and Ant + CD + CR $0.1 \%$ ) were infected by oral gavage with $10^{5}$ colony-forming units (CFU) per $0.1 \mathrm{ml}$ total volume of hypervirulent $C$. difficile spores (ATCC BAA 1805) using a straight 18 -gauge needle with 1 " shaft length, and were 
TABLE 1 | Different treatment groups used in the experiment.

\begin{tabular}{lccc}
\hline Treatment group $(\boldsymbol{n}=\mathbf{8}$ each) & Antibiotic & CR & C. difficile \\
\hline Negative control & - & - & - \\
Ant + CD & + & - & + \\
Ant + CR & + & $0.1 \%$ & - \\
CR control & - & $0.1 \%$ & - \\
Ant + CD + CR 0.05\% & + & $0.05 \%$ & + \\
Ant + CD + CR 0.1\% & + & $0.1 \%$ & + \\
Ant control & + & - & - \\
\hline
\end{tabular}

Ant, Antibiotic; CD, C. difficile; CR, Carvacrol.

monitored for signs of CDAD such as diarrhea, hunched posture and wet tail using a mouse clinical score sheet (Supplementary Table 1). Animals were observed twice daily for 10 days for mortality and morbidity. The individual weight of each animal was measured every day. Fecal samples from all animals were collected on alternate days post infection. The animals were euthanized at the end of the experiment (10th day after $C$. difficile challenge).

\section{DNA Extraction, PCR Amplification, and Sequencing of Taxonomic Marker}

DNA was extracted from $0.25 \mathrm{~g}$ of fecal sample (collected on the 2nd day post inoculation, DPI) from all treatment groups using the MoBio PowerMag Soil 96 well kit (MoBio Laboratories, Inc), according to the manufacturer's protocol for the Eppendorf ep Motion liquid handling robot. DNAquantification was performed using the Quant-iT PicoGreen kit (Invitrogen, ThermoFisher Scientific). Partial bacterial 16S rRNA genes (V4) were amplified using $30 \mathrm{ng}$ extracted DNA as template. The V4 region was amplified using $515 \mathrm{~F}$ and $806 \mathrm{R}$ with Illumina adapters and dual indices ( 8 basepair golay on 3'; Caporaso et al., 2012), and eight basepair on the $5^{\prime}$ (Kozich et al., 2013). Samples were amplified in triplicate using Phusion High-Fidelity PCR master mix (New England BioLabs) with the addition of $10 \mu \mathrm{g}$ BSA (New England BioLabs). The PCR reaction was incubated at $95^{\circ} \mathrm{C}$ for $3.5 \mathrm{~min}$, then 30 cycles of $30 \mathrm{~s}$ at $95.0^{\circ} \mathrm{C}, 30 \mathrm{~s}$ at $50.0^{\circ} \mathrm{C}$, and $90 \mathrm{~s}$ at $72.0^{\circ} \mathrm{C}$, followed by a final extension at $72.0^{\circ} \mathrm{C}$ for $1 \mathrm{~min}$ were used. The PCR products were pooled for quantification and visualization using the QIAxcel DNA Fast Analysis (Qiagen). The PCR products were normalized based on the concentration of DNA from 250 to $400 \mathrm{bp}$ and pooled using the QIAgility liquid handling robot. The pooled PCR products were cleaned using the Gene Read Size Selection kit (Qiagen) according to the manufacturer's protocol. The cleaned pool was sequenced on MiSeq using v2 $2 \times 250$ base pair kit (Illumina, Inc).

\section{Sequence Analysis}

The microbiome analysis was set up as a completely randomized design with treatments done in replicates of six. The sequences were filtered and clustered using Mothur 1.36.1 based on a published protocol with slight modifications (Kozich et al., 2013). Operational taxonomic units (OTUs) were clustered at $97 \%$ sequence similarity. Downstream analysis of samples was done using $\mathrm{R}$ version 3.2. The alpha diversity was calculated using inverse Simpson to measure the richness and evenness of the OTUs. The effect of treatment on the alpha diversity was analyzed using Tukey's Test. A permutational multivariate analysis (PERMANOVA, adonis function, 75 permutations) was conducted to analyze the effect of various treatments on the bacterial community composition. Significant change in alpha diversity was determined by Anova followed by Tukey's honest significant differences adjusting for multiple comparisons ( $p=$ $0.05)$. NMS ordinations were run in R (v 3.3.0) using metaMDS in the vegan (v 2.3-5) package after calculating the stress scree plots to determine the number of axes required to achieve stress below 0.2, plotted using ggplot2 ( $\mathrm{v}$ 2.1.0). Finally, the relative abundance of OTUs of major phyla, order, and genera was determined to assess the effect of treatment. Tukey's Test was used to identify changes in groups of bacteria based on treatment and the significance was detected at $P<0.05$.

\section{Statistical Analysis}

The results were expressed as means \pm standard errors of the means (SEM). The differences between the experimental groups were compared using the analysis of variance (ANOVA). Twoway ANOVA was used to compare experimental groups across the days. The differences between two groups were analyzed using unpaired Student's $t$-test. "N1" Chi-squared test were used to compare incidence rate between two different treatments. The statistical significance level was set at a $P<0.05$.

\section{RESULTS}

\section{Effect of CR Supplementation on the Incidence of Diarrhea and Severity of $C$. difficile Infection in Mice}

In order to assess the prophylactic effect of CR against $C$. difficile associated diarrhea in mice ( 8 mice per treatment group), the animal diets were supplemented with CR at two different concentrations $(0.05 \%$ and $0.1 \%)$ in feed prior to antibiotic treatment and subsequent $C$. difficile infection (Ant $+\mathrm{CD}+$ $0.05 \% \mathrm{CR}$ and Ant $+\mathrm{CD}+0.1 \%$ CRThe oral administration of $10^{5} \mathrm{CFU} / \mathrm{ml}$ NAP1 C. difficile spores resulted in high morbidity and low mortality in infected mice. In $C$. difficile infected control groups (Ant $+\mathrm{CD}), 75 \%(6 / 8)$ of the animals showed severe diarrhea on 1DPI, and $90 \%(7 / 8)$ of the animals showed severe diarrhea on 2DPI. Two animals died on 1DPI, and no further mortality was observed in this group. No increase in the incidence of diarrhea was observed after 2DPI (Figure 1). Interestingly, only $50 \%(4 / 8)$ of the animals showed diarrhea in $1 \mathrm{DPI}$ in Ant $+\mathrm{CD}+0.05 \%$ CR group (animals supplemented with $0.05 \% \mathrm{CR}$ prior to the antibiotic treatment and subsequent C. difficile infection), no increase in the incidence of diarrhea was reported after 1DPI. In Ant $+\mathrm{CD}+0.1 \%$ CR group, only $12.5 \%$ $(1 / 8)$ of the animals showed diarrhea on 1DPI, which further increased to $25 \%(2 / 8)$ on 2DPI and no further incidents were observed on subsequent days. In both CR-treated and C. difficile infected groups (Ant + CD + 0.05\% CR and Ant + CD + 0.1\% $\mathrm{CR}$ ), two mortalities each were recorded on 2DPI. No diarrheal 


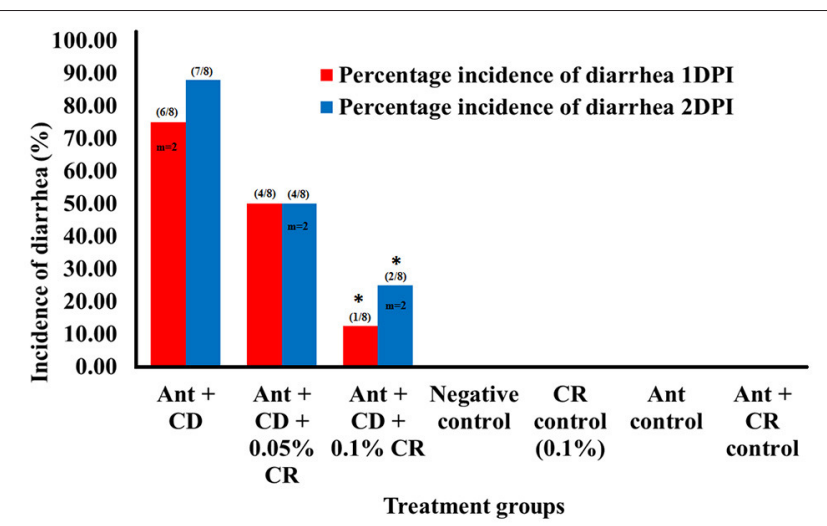

FIGURE 1 | Effect of CR supplementation on the incidence of $\mathbf{C}$. difficile associated diarrhea in mice. The incidence of diarrhea in different treatement groups was recorded after C. difficile challenge. Groups: (1) Negative Control: Mice treated with no CR, no antibiotics and no C. difficile (2) CR control: Mice fed with $0.1 \%$ CR in feed, (3) Ant Control: Mice administered with antibiotic cocktail in water and an intra-peritoneal injection of clindamycin, (4) Ant + CR Control: Mice fed with CR (0.1\%) supplemented feed and administered with antibiotic cocktail in water and an intra-peritoneal injection of clindamycin, (5) Ant + CD: administered with antibiotic cocktail in water and an intra-peritoneal injection of clindamycin, and infected by C. difficile (6) (Ant $+\mathrm{CD}+0.05 \% \mathrm{CR})$ : Mice fed with CR (0.05\%), administered with antibiotic cocktail in water and an intra-peritoneal injection of clindamycin, and infected with C. difficile) (Ant $+\mathrm{CD}+0.1 \% \mathrm{CR})$ : Mice fed with CR $(0.1 \%)$, administered with antibiotic cocktail in water and an intra-peritoneal injection of clindamycin, and infected with $C$. difficile. (' treatments significantly differed from infected control group (Ant $+\mathrm{CD}$ ) $p<0.05$; $\mathrm{m}$ indicates the number of mortalities recorded; cumulative incidence of diarrhea per total number of animals used in the experiment is shown in parenthesis).

symptoms were observed in control groups (Negative control, CR control, Ant control, and Ant + CR control).

\section{Effect of CR Supplementation on Clinical Score and Body Weight of C. difficile Infected Mice}

Clinical scores of the individual animals in different groups were recorded using standard clinical score chart, from 1DPI to 7 DPI (Chen et al., 2008; Sun et al., 2011). The C. difficile control group (Ant $+\mathrm{CD}$ group) exhibited significantly increased severity, as indicated by a higher average clinical score per group on 1, 2, 3 , and 4 DPI (Figure 2) $(p<0.05)$. The severity of CDAD in animals supplemented prophylactically with 0.05 and $0.1 \% \mathrm{CR}$ (Ant $+\mathrm{CD}+0.05 \% \mathrm{CR}$ and Ant $+\mathrm{CD}+0.1 \% \mathrm{CR}$ groups $)$ was lesser than that of the untreated group (Ant $+\mathrm{CD})(p<$ $0.05)$. On 1DPI, there was a dose-dependent reduction in the severity of infection in CR supplemented groups. Irrespective of the treatments, all surviving morbid animals recovered by 6DPI, as indicated by a zero clinical score.

A similar trend was observed in average body weights of animals in different treatment groups. Body weights were recorded daily and the relative percentage weight with respect to the initial weight before $C$. difficile infection was calculated (Figure 3). Carvacrol alone (CR control) and in combination with antibiotic (Ant $+\mathrm{CR}$ control) did not cause any significant

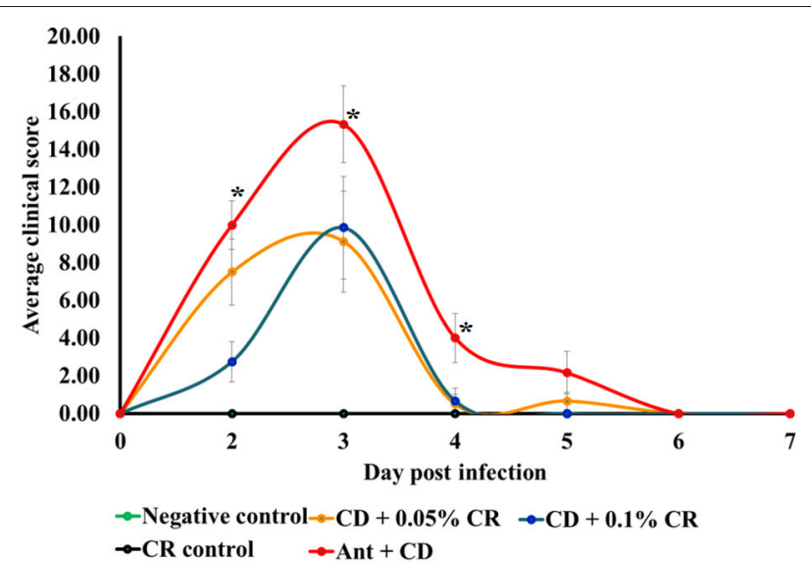

FIGURE 2 | Effect of CR supplementation on the severity of C. difficile associated disease in mice. The severeity of $C$. difficile associated disease in different treatment groups was determined based on a clinical score sheet. Groups: (1) Negative Control: Mice treated with no CR, no antibiotics and no C. difficile (2) CR control: Mice fed with $0.1 \%$ CR in feed, (3) Ant Control: Mice administered with antibiotic cocktail in water and an intra-peritoneal injection of clindamycin, (4) Ant + CR Control: Mice fed with CR (0.1\%) supplemented feed and administered with antibiotic cocktail in water and an intra-peritoneal injection of clindamycin, (5) Ant + CD: administered with antibiotic cocktail in water and an intra-peritoneal injection of clindamycin, and infected by $C$. difficile 6) (Ant + CD + 0.05\% CR): Mice fed with CR (0.05\%), administered with antibiotic cocktail in water and an intra-peritoneal injection of clindamycin, and infected with C. difficile) (Ant + CD + 0.1\% CR): Mice fed with CR $(0.1 \%)$, administered with antibiotic cocktail in water and an intra-peritoneal injection of clindamycin, and infected with $C$. difficile. ( ${ }^{*}$ The clinincal scores of positive control group (Ant $+C D$ were significantly greater than that of Ant $+C D+C R$ $0.1 \%$ and control groups, $p<0.05$ ).

weight loss compared to the negative control. All mice in the C. difficile control group (Ant $+\mathrm{CD}$ ) showed significant and progressive weight loss from 1 DPI to 5DPI compared to the negative control $(p<0.05)$. However, CR-treated and $C$. difficile infected mice (Ant $+\mathrm{CD}+0.05 \% \mathrm{CR}$ and Ant $+\mathrm{CD}+0.1 \% \mathrm{CR}$ groups) showed a significantly lesser weight loss in comparison to untreated and $C$. difficile infected group (Ant $+\mathrm{CD})$ from $1 \mathrm{DPI}$ and 2DPI, with regaining of the initial weight on 3DPI $(p<0.05)$. No significant difference in recorded weight loss was observed between 0.05 and $0.1 \%$ CR-treated C. difficile infected mice except for a rapid and early increase in the body weight on 2DPI in the $0.05 \%$ CR group.

\section{Effect of CR Supplementation on the Gut Microbiome of C. difficile Infected and Non-infected mice}

Microbiome analysis results revealed specific patterns in the composition of different bacterial taxa in different treatment groups. In the phylum level, the gut microbiome of negative control mice was predominated by Bacteriodetes, followed by Firmicutes (Figure 4) with a minimal proportion of other phyla, including Proteobacteria. A similar trend was observed in CR control group, with an abundance of Bacteriodetes followed by Firmicutes, although the proportion of Firmicutes 


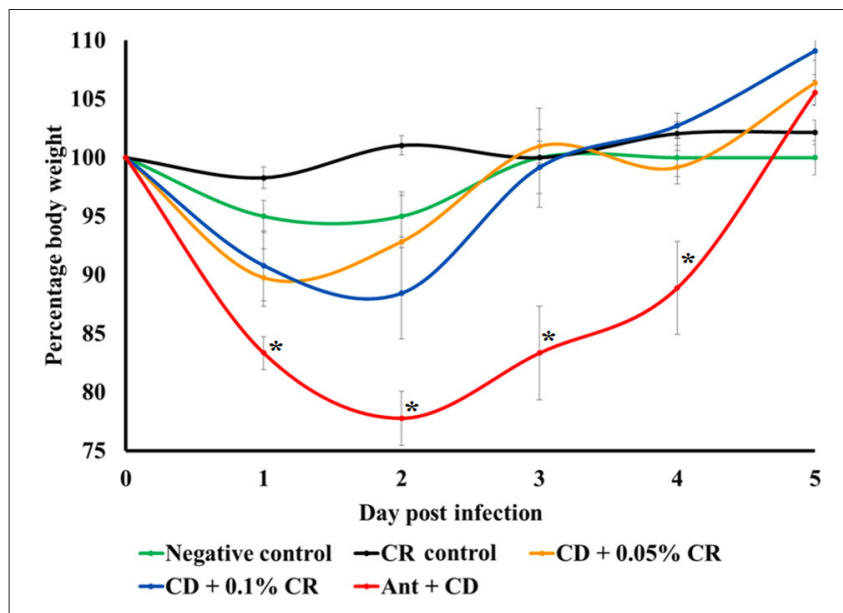

FIGURE 3 | Effect of CR supplementation on relative weight loss in C. difficile infected and non-infected mice. The body weights of the animals were recorded daily and the relative percentage weight with respect to the initial weight prior to the infection was calculated. Groups: (1) Negative Control: Mice treated with no CR, no antibiotics and no C. difficile (2) CR control: Mice fed with $0.1 \% \mathrm{CR}$ in feed, (3) Ant Control: Mice administered with antibiotic cocktail in water and an intra-peritoneal injection of clindamycin, (4) Ant + CR Control: Mice fed with CR $(0.1 \%)$ supplemented feed and administered with antibiotic cocktail in water and an intra-peritoneal injection of clindamycin, (5) Ant + CD: administered with antibiotic cocktail in water and an intra-peritoneal injection of clindamycin, and infected by C. difficile (6) (Ant + CD + 0.05\% $\mathrm{CR})$ : Mice fed with CR (0.05\%), administered with antibiotic cocktail in water and an intra-peritoneal injection of clindamycin, and infected with $C$. difficile) (Ant + CD $+0.1 \%$ CR): Mice fed with CR $(0.1 \%)$, administered with antibiotic cocktail in water and an intra-peritoneal injection of clindamycin, and infected with $C$. difficile. ( ${ }^{*}$ The relative weight loss of positive control group (Ant $+\mathrm{CR}$ ) was significantly greater than Ant + CD + CR 0.05\%, Ant + CD + CR 0.1\% and control groups, $p<0.05)$.

was slightly higher than that of the negative control group. Antibiotic administration significantly increased the proportion of Proteobacteria in antibiotic-treated (Ant control) group compared to the negative control and CR control groups $(p<$ 0.05). Interestingly, supplementation of $C R$ along with antibiotic (Ant + CR control) significantly reduced the abundance of Proteobacteria compared to the antibiotic only (Ant control) group. The $C$. difficile control group (Ant $+\mathrm{CD})$, where C. difficile spores were orally gavaged after antibiotic treatment, exhibited a remarkably increased abundance of Proteobacteria along with a greater proportion of Verrucomicrobia compared to all other control groups (Negative control, CR control, Ant control, Ant + CR control groups $)(p<0.05)$. In addition, the abundance of Bacteriodetes and Firmicutes was significantly reduced in $C$. difficle control group (Ant $+\mathrm{CD})$, compared to uninfected controls $(p<0.05)$. Strikingly, this alteration in the abundance of Proteobacteria, Firmicutes, Bacteriodetes and Verrucobacteria due to $C$. difficile infection was reversed significantly by CR supplementation, as observed in the CRtreated and C. difficile infected groups (Ant + CD + 0.05\% CR and Ant $+\mathrm{CD}+0.10 \%$ CR groups $)(p<0.05)$.

At the Order and Family level, an increased abundance of Enterobacteriaceae was observed in antibiotic alone (Ant

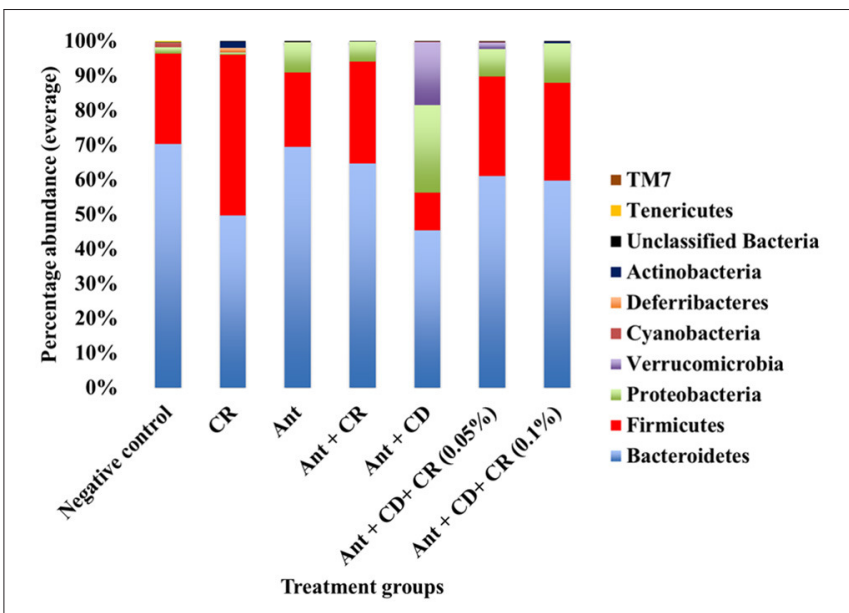

FIGURE 4 | Effect of CR supplementation on the abundance of major gut microbiota (phyla level) in the antibiotic treated and C. difficile challenged mice. The relative abundance of OTUs at different taxonomic levels was determined by gut microbiome analysis. Groups: (1) Negative Control: Mice treated with no $\mathrm{CR}$, no antibiotics and no C. difficile (2) CR control: Mice fed with $0.1 \%$ CR in feed, (3) Ant Control: Mice administered with antibiotic cocktail in water and an intra-peritoneal injection of clindamycin, (4) Ant + CR Control: Mice fed with CR $(0.1 \%)$ supplemented feed and administered with antibiotic cocktail in water and an intra-peritoneal injection of clindamycin, (5) Ant + CD: administered with antibiotic cocktail in water and an intra-peritoneal injection of clindamycin, and infected by C. difficile (6) (Ant + CD + 0.05\% CR): Mice fed with CR (0.05\%), administered with antibiotic cocktail in water and an intra-peritoneal injection of clindamycin, and infected with $C$. difficile) $($ Ant $+C D+0.1 \% C R)$ : Mice fed with $C R(0.1 \%)$, administered with antibiotic cocktail in water and an intra-peritoneal injection of clindamycin, and infected with C. difficile.

control) and C. difficile (Ant $+\mathrm{CD}$ ) groups compared to negative control and CR control groups (Figure 5). Moreover, CR supplementation significantly reduced the abundance of Enterobacteriaceae induced by the antibiotic administration and C. difficile infection, as indicated by a significant reduction in their abundance in Ant + CR control group, Ant $+C D+0.05 \%$ $\mathrm{CR}$, and Ant $+\mathrm{CD}+0.10 \%$ CR groups $(p<0.05)$ (Figure 5). Carvacrol treatment significantly increased the abundance of Lactobacillaceae and Lachnospiraceae in the gut microbiome compared to that of negative control (Figure 5) $(p<0.05)$. The abundance of Lactobacillaceae and Lachnospiraceae was significantly reduced following antibiotic treatment (Ant control group) and $C$. difficile (Ant $+\mathrm{CD}$ control group) infection, compared to the Negative control and CR alone (CR control) groups $(p<0.05)$. This effect was significantly reversed by the supplementation of CR in Ant + CR control, Ant $+\mathrm{CD}+0.5$ $\mathrm{CR}$, and Ant $+\mathrm{CD}+0.5 \mathrm{CR}$ groups.

Inverse Simpson plot revealed a differential pattern of bacterial diversity in various treatment groups (Figure 6). Strikingly, CR treatment did not alter the diversity of the gut bacterial community compared to the untreated control group (Negative control). As expected, antibiotic treatment significantly reduced the bacterial diversity compared to control and CR group. There was a marked reduction in the diversity of the bacterial community in $C$. difficile infected groups, irrespective 


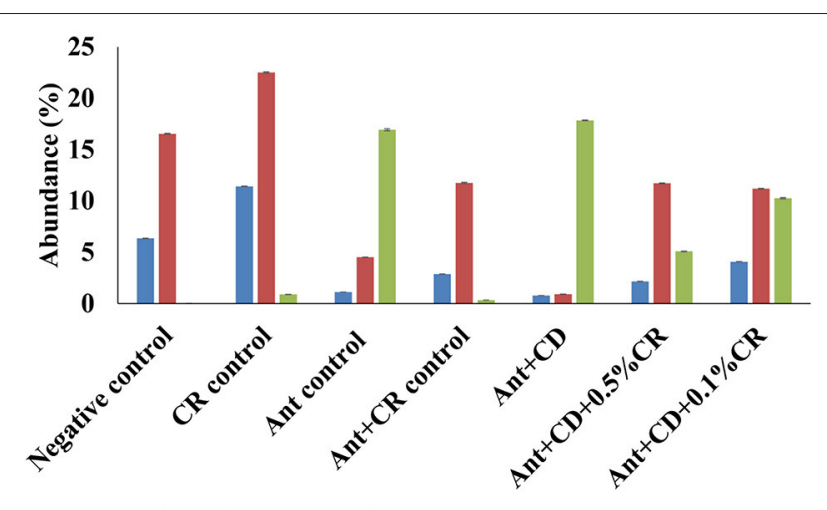

- Lactobacillaceae $\square$ Lachnospiraceae Enterobacteriaceae

FIGURE 5 | Effect of CR supplementation on the abundance of Enterobacteriaceae, Lactobacillaceae and Lachnospiraceae in the antibiotic treated and $\boldsymbol{C}$. difficile challenged mice. The relative abundance of OTUs at family level (Family. Enterobacteriaceae, Lactobacillaceae and Lachnospraceae) was by determined using gut microbiome analysis. Groups: (1) Negative Control: Mice treated with no CR, no antibiotics and no C. difficile (2) CR control: Mice fed with $0.1 \%$ CR in feed, (3) Ant Control: Mice administered with antibiotic cocktail in water and an intra-peritoneal injection of clindamycin, (4) Ant + CR Control: Mice fed with CR (0.1\%) supplemented feed and administered with antibiotic cocktail in water and an intra-peritoneal injection of clindamycin, (5) Ant + CD: administered with antibiotic cocktail in water and an intra-peritoneal injection of clindamycin, and infected by $C$. difficile (6) (Ant + CD + 0.05\% CR): Mice fed with CR (0.05\%), administered with antibiotic cocktail in water and an intra-peritoneal injection of clindamycin, and infected with C. difficile (7) (Ant $+\mathrm{CD}+0.1 \% \mathrm{CR})$ : Mice fed with CR $(0.1 \%)$, administered with antibiotic cocktail in water and an intra-peritoneal injection of clindamycin, and infected with $C$. difficile. (Treatments significantly differed from respective control groups, $p<0.05)$.

of the CR treatment. Moreover, NMDS plot representing the relationships between samples in various treatment groups based on the abundance of species present in each sample revealed a close clustering of CR control samples and untreated control samples (Figure 7). This representation suggests that the species abundance in CR treatment groups is comparable to untreated mice indicating minimal effect of CR on gut microbial diversity.

\section{DISCUSSION}

Prolonged antibiotic therapy and subsequent gut dysbiosis result in $C$. difficile spore germination, and colonization of the large intestine with vegetative cells of the bacterium, and subsequent production of toxins TcdA and TcdB, resulting in C. difficile associated diarrhea (Hookman and Barkin, 2009). C. difficile toxins lead to intestinal inflammation, increased epithelial permeability (Castagliuolo et al., 1998; Feltis et al., 2000; He et al., 2002), enhanced cytokine and chemokine production (Castagliuolo et al., 1998; He et al., 2002), neutrophil infiltration (Kelly et al., 1994) and the release of reactive oxygen intermediates (He et al., 2002), thereby causing direct damage to the intestinal mucosa ( $\mathrm{Ng}$ et al., 2010). Antibiotics are the primary line of treatment in C. difficile infection, although the use of antibiotics has been documented for inducing and aggravating gut dysbiosis and relapse of the infection post-therapy. In

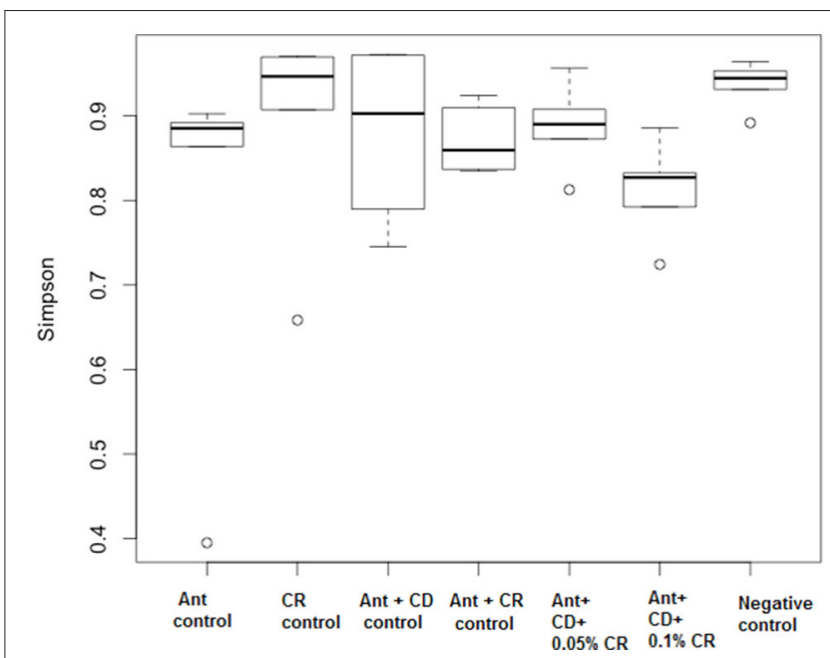

FIGURE 6 | Effect of CR supplementation on the diversity of gut microbiota of antibiotic treated and C. difficile challenged mice. Alpha diversity of the gut microbiome was calculated by using inverse Simpson to measure the richness and evenness of the OTUs. Groups: (1) Negative Control: Mice treated with no CR, no antibiotics and no C. difficile (2) CR control: Mice fed with $0.1 \% \mathrm{CR}$ in feed, (3) Ant Control: Mice administered with antibiotic cocktail in water and an intra-peritoneal injection of clindamycin, (4) Ant + CR Control: Mice fed with CR (0.1\%) supplemented feed and administered with antibiotic cocktail in water and an intra-peritoneal injection of clindamycin, (5) Ant + CD: administered with antibiotic cocktail in water and an intra-peritoneal injection of clindamycin, and infected by C. difficile (6) (Ant $+\mathrm{CD}+0.05 \% \mathrm{CR})$ : Mice fed with CR $(0.05 \%)$, administered with antibiotic cocktail in water and an intra-peritoneal injection of clindamycin, and infected with C. difficile (7) (Ant + CD + 0.1\% CR): Mice fed with CR $(0.1 \%)$, administered with antibiotic cocktail in water and an intra-peritoneal injection of clindamycin, and infected with C. difficile.

addition, increasing incidence of $C$. difficile acquiring antibiotic resistance is reported worldwide.

In the current study, we investigated the prophylactic efficacy of $\mathrm{CR}$ as an alternative antimicrobial agent that can ameliorate C. difficile associated diarrhea without inducing gut dysbiosis. Previous studies have reported that supplementation of low doses of CR exerted no detrimental effects on endogenous bacterial populations, including Lactobacilli and Bifidobacteria in pigs and poultry (Jamroz et al., 2005; Si et al., 2006).

Previous studies conducted in our laboratory revealed that SICs of CR reduced C. difficile toxin production and cytotoxicity in Vero cells in vitro (Mooyottu et al., 2014a). In addition, our previous experiments showed an inhibition of $C$. difficile spore outgrowth in the presence of CR (Mooyottu et al., 2014b). The results from the mice experiment indicated that our in vitro results apparently well translated in vivo with regards to the clinical outcome and gut microbiome of the animals when prophylactically treated with $\mathrm{CR}$ prior to $C$. difficile infection. As expected, CR supplementation significantly reduced the incidence of diarrhea in $C$. difficile infected mice $(p<$ 0.05). Moreover, CR supplementation significantly reduced the severity of clinical infection in $C$. difficile infected mice, as evident from a reduced average clinical score compared to the 


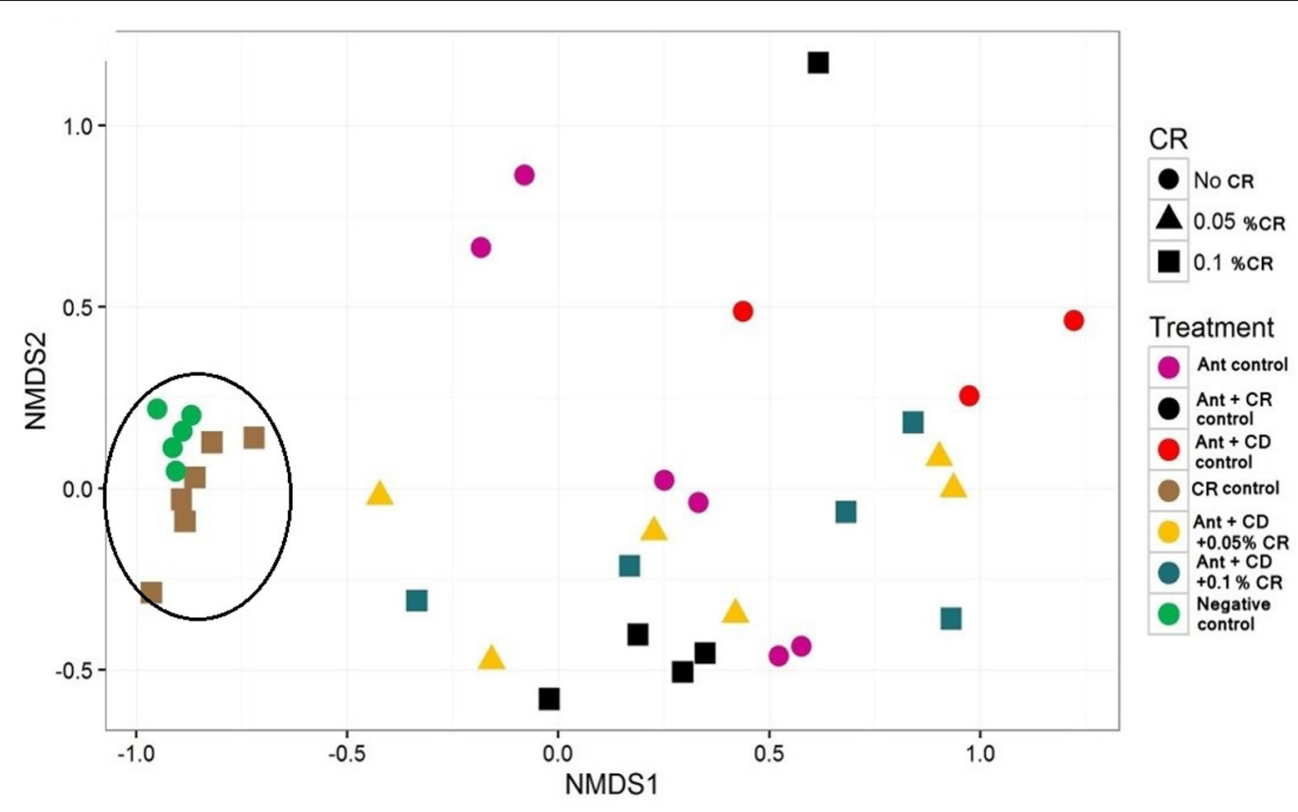

FIGURE 7 | Effect of CR supplementation on the diversity of gut microbiota of antibiotic treated and C. difficile challenged mice. Relationships between treatment groups based on the abundance of species present in each sample were plotted. Groups: (1) Negative Control: Mice treated with no CR, no antibiotics and no C. difficile (2) CR Control: Mice fed with 0.1\% CR in feed, (3) Ant Control: Mice administered with antibiotic cocktail in water and an intra-peritoneal injection of clindamycin, (4) Ant + CR Control: Mice fed with CR (0.1\%) supplemented feed and administered with antibiotic cocktail in water and an intra-peritoneal injection of clindamycin, (5) Ant + CD: administered with antibiotic cocktail in water and an intra-peritoneal injection of clindamycin, and infected by C. difficile (6) (Ant + CD + $0.05 \% \mathrm{CR})$ : Mice fed with CR (0.05\%), administered with antibiotic cocktail in water and an intra-peritoneal injection of clindamycin, and infected with C. difficile (7) (Ant $+\mathrm{CD}+0.1 \% \mathrm{CR})$ : Mice fed with $\mathrm{CR}(0.1 \%)$, administered with antibiotic cocktail in water and an intra-peritoneal injection of clindamycin, and infected with $\mathrm{C}$. difficile. Circle indicates close clustering of CR Control samples and Nagative Control samples.

infected control group (Ant + CD) $(p<0.05)$. However, CRtreated and $C$. difficile infected mice exhibited significantly lesser weight loss compared to the untreated group (Ant $+\mathrm{CD}$ ). The reduced severity of CDAD in CR-treated mice compared to infected control group (Ant $+\mathrm{CD}$ ) could be attributed to an inhibitory effect of the phytochemical on $C$. difficile spore outgrowth and/or the toxin production, as observed in our in vitro studies. Moreover, CR has been reported to possess antiinflammatory and anti-diarrheal properties (Baser, 2008), which could also have exerted a beneficial effect in $C$. difficile infected mice.

A healthy and normal gut microflora is crucial for preventing pathogen colonization and a variety of enteric bacterial infections, including C. difficile (Britton and Young, 2014). The most important predisposing factor for $C$. difficile infection is the disruption of normal gut microbiota (Hookman and Barkin, 2009). Antibiotic therapy significantly alters the microbial composition and diversity; and in many cases alterations in the microbial diversity persist to an extent, even after withdrawing antibiotic administration (Dethlefsen et al., 2008; Antonopoulos et al., 2009). In human patients, as age advances, the protective bacterial population of Firmicutes considerably diminishes accompanied by an increase in Bacteroidetes and undesirable species of Proteobacteria in the gut (Hopkins et al., 2001; Biagi et al., 2010; Claesson et al., 2011). Moreover, agerelated senescence in the immune status of the elderly, along with frequent hospital visits during old age contribute to a detrimental alteration in the gut microbiome and subsequent colonization of C. difficile (Seekatz and Young, 2014). Other important factors that detrimentally affect the gut microbiota and predispose $C$. difficile infection are the use of proton pump inhibitors and chronic gastrointestinal diseases (Dial et al., 2005; Vesper et al., 2009; Berg et al., 2013). Proton pump inhibitors alter the $\mathrm{pH}$ of the gut, thereby affecting the microbial population, especially beneficial bacteria such as Lactobacillus species (Altman et al., 2008; Vesper et al., 2009). In addition, disease conditions such as inflammatory bowel disease (IBD) induce significant gut dysbiosis, which reduces the diversity of the protective population of Firmicutes and Bacteroides population accompanied by an increased Proteobacteria in the gut of affected patients (Manichanh et al., 2006; Nagalingam and Lynch, 2012). Increased abundance of Verrucomicrobia has also been shown in patients with antibiotic-associated gut dysbiosis (Weingarden et al., 2014). Moreover, the paucity of Firmicutes, especially the depletion of Ruminococcaceae, Lachnospiraceae, and butyrogenic bacteria within this phylum observed in $C$. difficile infection and nosocomial diarrhea in humans (Antharam et al., 2013). Moreover, C. difficile infected patients have a higher count of Enterobacteriaceae (Proteobacteria) and decreased Enterococcaceae (Firmicutes) (Hopkins and Macfarlane, 2002; Rea et al., 2012; Schubert et al., 2014). 
The changes in the gut microbiome diversity and alterations in the relative abundance of different bacterial communities in human patients are replicated in mice models of $C$. difficile infection (Semenyuk et al., 2015). Antibiotic treatment and subsequent $C$. difficile infection significantly reduced the abundance of Firmicutes and Bacteroides in phylum level. Similar trends are observed in all taxonomical level such as a reduction in protective Lactobacillaceae, Lachnospiracea, and Bifidobacteriacea. A dramatic increase in the abundance of Proteobacteria specifically Enterobacteriaceae has been found in the antibiotic treated and $C$. difficile infected mice (Semenyuk et al., 2015). Similarly, antibiotic treatment and subsequent C. difficile infection significantly reduced microbiome diversity in mouse gut (Semenyuk et al., 2015).

In this study, CR treatment did not reduce the bacterial diversity in the mouse gut. To date, a majority of the antimicrobial compounds, especially antibiotics, have significantly altered the microbial diversity, and cause dysbiosis by changing the abundance of bacterial communities (Semenyuk et al., 2015). Moreover, CR treatment significantly increased the abundance of beneficial bacterial populations such as Firmicutes, specifically the members of Lactobacillaceae and Lachnospiraceae. In addition, CR treatment alone did not increase the abundance of detrimental bacterial populations compared to untreated control animals. Strikingly, CR reduced antibiotic-induced increases in the abundance of unfavorable bacterial populations such as Proteobacteria, specifically pathogenic gamma proteobacteria, including Enterobacteriaceae and other bacterial populations such as Verrucobacteria (Figure 5). Surprisingly, this beneficial shift brought about by CR treatment in the gut microbiome of antibiotic-treated and $C$. difficile infected animals is very much similar to that of human patients who have undergone fecal microbiome transplantation (Weingarden et al., 2014), which is documented as one of the

\section{REFERENCES}

Altman, K. W., Chhaya, V., Hammer, N. D., Pavlova, S., Vesper, B. J., Tao, L., et al. (2008). Effect of proton pump inhibitor pantoprazole on growth and morphology of oral Lactobacillus strains. Laryngoscope 118, 599-604. doi: 10.1097/MLG.0b013e31816 1f9bf

Antharam, V. C., Li, E. C., Ishmael, A., Sharma, A., Mai, V., Rand, K. H., et al. (2013). Intestinal dysbiosis and depletion of butyrogenic bacteria in Clostridium difficile infection and nosocomial diarrhea. J. Clin. Microbiol. 51, 2884-2892. doi: 10.1128/JCM.00845-13

Antonopoulos, D. A., Huse, S. M., Morrison, H. G., Schmidt, T. M., Sogin, M. L., and Young, V. B. (2009). Reproducible community dynamics of the gastrointestinal microbiota following antibiotic perturbation. Infect. Immun. 77, 2367-2375. doi: 10.1128/IAI.01520-08

Bartlett, J. G. (1992). Antibiotic-associated diarrhea. Clin. Infect. Dis. 15, 573-581.

Baser, K. H. (2008). Biological and pharmacological activities of carvacrol and carvacrol bearing essential oils. Curr. Pharm. Des. 14, 3106-3119. doi: 10.2174/138161208786404227

Berg, A. M., Kelly, C. P., and Farraye, F. A. (2013). Clostridium difficile infection in the inflammatory bowel disease patient. Inflamm. Bowel Dis. 19, 194-204. doi: $10.1002 /$ ibd.22964

Biagi, E., Nylund, L., Candela, M., Ostan, R., Bucci, L., Pini, E., et al. (2010). Through ageing, and beyond: gut microbiota and inflammatory status in seniors and centenarians. PLOS ONE 5:e10667. doi: 10.1371/journal.pone.0010667 most effective strategies against severe $C$. difficile infection (Schenck et al., 2015; Ofosu, 2016). These results suggest that reduced or delayed clinical infection rate and less severe clinical presentation of CR-treated animals could attributed in part to the beneficial shift in the gut microbiome.

To conclude, our results suggest CR supplementation to be protective against $C$. difficile infection in mice. Carvacrol supplementation significantly reduced the incidence of diarrhea and mitigated the severity of $C$. difficile induced clinical symptoms, inducing a favorable shift in the composition of the gut microbiota without detrimentally affecting the gut microbiome diversity in mice. These findings suggest the potential of $\mathrm{CR}$ as an anti- $C$. difficile agent, however, further clinical studies are warranted to confirm this.

\section{AUTHOR CONTRIBUTIONS}

$\mathrm{KV}$ conceived the idea, $\mathrm{KV}$ and $\mathrm{SM}$ designed the experiments and prepared the manuscripts, SM, GF, and AU performed the experiments, $\mathrm{KM}$ and IU analyzed the data.

\section{ACKNOWLEDGMENTS}

This research was funded by United States Department of Agriculture-National Institute of Food and Agriculture (USDANIFA) Critical and Emerging Food Safety Issues program Grant \# 2010-03567.

\section{SUPPLEMENTARY MATERIAL}

The Supplementary Material for this article can be found online at: http://journal.frontiersin.org/article/10.3389/fmicb. 2017.00625/full\#supplementary-material

Blossom, D. B., and McDonald, L. C. (2007). The challenges posed by reemerging Clostridium difficile infection. Clin. Infect. Dis. 45, 222-227. doi: $10.1086 / 518874$

Britton, R. A., and Young, V. B. (2014). Role of the intestinal microbiota in resistance to colonization by Clostridium difficile. Gastroenterology 146, 1547-1553. doi: 10.1053/j.gastro.2014.01.059

Caporaso, J. G., Lauber, C. L., Walters, W. A., Berg-Lyons, D., Huntley, J., Fierer, N., et al. (2012). Ultra-high-throughput microbial community analysis on the Illumina HiSeq and MiSeq platforms. ISME J. 6, 1621-1624. doi: 10.1038/ismej. 2012.8

Castagliuolo, I., Keates, A. C., Wang, C. C., Pasha, A., Valenick, L., Kelly, C. P., et al. (1998). Clostridium difficile toxin A stimulates macrophageinflammatory protein-2 production in rat intestinal epithelial cells. J. Immunol. 160, 6039-6045.

Chen, X., Katchar, K., Goldsmith, J. D., Nanthakumar, N., Cheknis, A., Gerding, D. N., et al. (2008). A mouse model of Clostridium difficile-associated disease. Gastroenterology 135, 1984-1992. doi: 10.1053/j.gastro.2008.09.002

Claesson, M. J., Cusack, S., O’Sullivan, O., Greene-Diniz, R., de Weerd, H., Flannery, E., et al. (2011). Composition, variability, and temporal stability of the intestinal microbiota of the elderly. Proc. Natl. Acad. Sci. U.S.A. 108(Suppl. 1), 4586-4591. doi: 10.1073/pnas.1000097107

Dethlefsen, L., Huse, S., Sogin, M. L., and Relman, D. A. (2008). The pervasive effects of an antibiotic on the human gut microbiota, as revealed by deep 16S rRNA sequencing. PLoS Biol. 6:e280. doi: 10.1371/journal.pbio.00 60280 
Dial, S., Delaney, J. A., Barkun, A. N., and Suissa, S. (2005). Use of gastric acidsuppressive agents and the risk of community-acquired Clostridium difficileassociated disease. JAMA 294, 2989-2995. doi: 10.1001/jama.294.23.2989

Feltis, B. A., Wiesner, S. M., Kim, A. S., Erlandsen, S. L., Lyerly, D. L., Wilkins, T. D., et al. (2000). Clostridium difficile toxins A and B can alter epithelial permeability and promote bacterial paracellular migration through HT-29 enterocytes. Shock 14, 629-634. doi: 10.1097/00024382-200014060-00010

Ghose, C., Kalsy, A., Sheikh, A., Rollenhagen, J., John, M., Young, J., et al. (2007). Transcutaneous immunization with Clostridium difficile toxoid A induces systemic and mucosal immune responses and toxin A-neutralizing antibodies in mice. Infect. Immun. 75, 2826-2832. doi: 10.1128/IAI.00127-07

He, D., Sougioultzis, S., Hagen, S., Liu, J., Keates, S., Keates, A. C., et al. (2002). Clostridium difficile toxin A triggers human colonocyte IL-8 release via mitochondrial oxygen radical generation. Gastroenterology 122, 1048-1057. doi: 10.1053 /gast.2002.32386

Hookman, P., and Barkin, J. S. (2009). Clostridium difficile associated infection, diarrhea and colitis. World J. Gastroenterol. 15, 1554-1580. doi: 10.3748 /wjg. 15.1554

Hopkins, M. J., and Macfarlane, G. T. (2002). Changes in predominant bacterial populations in human faeces with age and with Clostridium difficile infection. J. Med. Microbiol. 51, 448-454. doi: 10.1099/0022-1317-51-5-448

Hopkins, M. J., Sharp, R., and Macfarlane, G. T. (2001). Age and disease related changes in intestinal bacterial populations assessed by cell culture, $16 \mathrm{~S}$ rRNA abundance, and community cellular fatty acid profiles. Gut 48, 198-205. doi: 10.1136/gut.48.2.198

Jamroz, D., Wiliczkiewicz, A., Wertelecki, T., Orda, J., and Skorupinska, J. (2005). Use of active substances of plant origin in chicken diets based on maize and locally grown cereals. Br. Poult. Sci. 46, 485-493. doi: 10.1080/00071660500191056

Kassam, Z., Lee, C. H., Yuan, Y., and Hunt, R. H. (2013). Fecal microbiota transplantation for Clostridium difficile infection: systematic review and meta-analysis. Am. J. Gastroenterol. 108, 500-508. doi: 10.1038/ajg. 2013.59

Keel, M. K., and Songer, J. G. (2006). The comparative pathology of Clostridium difficile-associated disease. Vet. Pathol. 43, 225-240. doi: 10.1354/vp.43-3-225

Kelly, C. P., Becker, S., Linevsky, J. K., Joshi, M. A., O’Keane, J. C., Dickey, B. F., et al. (1994). Neutrophil recruitment in Clostridium difficile toxin A enteritis in the rabbit. J. Clin. Invest. 93, 1257-1265. doi: 10.1172/JCI117080

Kelly, C. P., and LaMont, J. T. (1998). Clostridium difficile infection. Annu. Rev. Med. 49, 375-390. doi: 10.1146/annurev.med.49.1.375

Kozich, J. J., Westcott, S. L., Baxter, N. T., Highlander, S. K., and Schloss, P. D. (2013). Development of a dual-index sequencing strategy and curation pipeline for analyzing amplicon sequence data on the MiSeq Illumina sequencing platform. Appl. Environ. Microbiol. 79, 5112-5120. doi: 10.1128/AEM.01 043-13

Ling, Z., Liu, X., Jia, X., Cheng, Y., Luo, Y., Yuan, L., et al. (2014). Impacts of infection with different toxigenic Clostridium difficile strains on faecal microbiota in children. Sci. Rep. 4:7485. doi: 10.1038/srep07485

Manichanh, C., Rigottier-Gois, L., Bonnaud, E., Gloux, K., Pelletier, E., Frangeul, L., et al. (2006). Reduced diversity of faecal microbiota in Crohn's disease revealed by a metagenomic approach. Gut 55, 205-211. doi: 10.1136/gut.2005.073817

McDonald, L. C., Owings, M., and Jernigan, D. B. (2006). Clostridium difficile infection in patients discharged from US short-stay hospitals, 1996-2003. Emerging Infect. Dis. 12, 409-415. doi: 10.3201/eid1205. 051064

McFarland, L. V. (2008). Antibiotic-associated diarrhea: epidemiology, trends and treatment. Future Microbiol. 3, 563-578. doi: 10.2217/17460913.3.5.563

Mooyottu, S., Flock, G., and Venkitanarayanan, K. (2014b). "Carvacrol reduces spore production in hypervirulent Clostridium difficile," in Anonymous 114th General Meeting, American Society for Microbiology, (Boston, MA).

Mooyottu, S., Kollanoor-Johny, A., Flock, G., Bouillaut, L., Upadhyay, A., Sonenshein, A. L., et al. (2014a). Carvacrol and trans-cinnamaldehyde reduce Clostridium difficile toxin production and cytotoxicity in vitro. Int. J. Mol. Sci. 15, 4415-4430. doi: 10.3390/ijms15034415

Nagalingam, N. A., and Lynch, S. V. (2012). Role of the microbiota in inflammatory bowel diseases. Inflamm. Bowel Dis. 18, 968-984. doi: 10.1002/ibd.21866
Ng, J., Hirota, S. A., Gross, O., Li, Y., Ulke-Lemee, A., Potentier, M. S., et al. (2010). Clostridium difficile toxin-induced inflammation and intestinal injury are mediated by the inflammasome. Gastroenterology 139, 542-552. doi: 10.1053/j.gastro.2010.04.005

O'Connor, K. A., Kingston, M., O’Donovan, M., Cryan, B., Twomey, C., and O’Mahony, D. (2004). Antibiotic prescribing policy and Clostridium difficile diarrhoea. QJM 97, 423-429. doi: 10.1093/qjmed/hch076

Ofosu, A. (2016). Clostridium difficile infection: a review of current and emerging therapies. Ann. Gastroenterol. 29, 147-154. doi: 10.20524/aog.2016. 0006

Rea, M. C., O’Sullivan, O., Shanahan, F., O’Toole, P. W., Stanton, C., Ross, R. P., et al. (2012). Clostridium difficile carriage in elderly subjects and associated changes in the intestinal microbiota. J. Clin. Microbiol. 50, 867-875. doi: 10.1128/JCM.05176-11

Schenck, L. P., Beck, P. L., and MacDonald, J. A. (2015). Gastrointestinal dysbiosis and the use of fecal microbial transplantation in Clostridium difficile infection. World J. Gastrointest. Pathophysiol. 6, 169-180. doi: 10.4291/wjgp.v6.i4.169

Schubert, A. M., Rogers, M. A., Ring, C., Mogle, J., Petrosino, J. P., Young, V. B., et al. (2014). Microbiome data distinguish patients with Clostridium difficile infection and non-C. difficile-associated diarrhea from healthy controls. MBio 5, e01021-e01014. doi: 10.1128/mBio.01021-14

Seekatz, A. M., and Young, V. B. (2014). Clostridium difficile and the microbiota. J. Clin. Invest. 124, 4182-4189. doi: 10.1172/JCI 72336

Semenyuk, E. G., Poroyko, V. A., Johnston, P. F., Jones, S. E., Knight, K. L., Gerding, D. N., et al. (2015). Analysis of bacterial communities during Clostridium difficile infection in the mouse. Infect. Immun. 83, 4383-4391. doi: 10.1128/IAI.00145-15

Shah, D., Dang, M. D., Hasbun, R., Koo, H. L., Jiang, Z. D., DuPont, H. L., et al. (2010). Clostridium difficile infection: update on emerging antibiotic treatment options and antibiotic resistance. Expert Rev. Anti Infect. Ther. 8, 555-564. doi: $10.1586 /$ eri.10.28

Shahinas, D., Silverman, M., Sittler, T., Chiu, C., Kim, P., Allen-Vercoe, E., et al. (2012). Toward an understanding of changes in diversity associated with fecal microbiome transplantation based on 16S rRNA gene deep sequencing. MBio 3:e00338-12. doi: 10.1128/mBio.00338-12

Si, W., Gong, J., Chanas, C., Cui, S., Yu, H., Caballero, C., et al. (2006). In vitro assessment of antimicrobial activity of carvacrol, thymol and cinnamaldehyde towards Salmonella serotype Typhimurium DT104: effects of pig diets and emulsification in hydrocolloids. J. Appl. Microbiol. 101, 1282-1291. doi: 10.1111/j.1365-2672.2006.03045.x

Spigaglia, P., Barbanti, F., Mastrantonio, P., and European Study Group on Clostridium difficile (ESGCD). (2011). Multidrug resistance in European Clostridium difficile clinical isolates. J. Antimicrob. Chemother. 66, 2227-2234. doi: $10.1093 / \mathrm{jac} / \mathrm{dkr} 292$

Steiner, C., Barrett, M., and Terrel, L. (2012). HCUP projections: Clostridium difficile hospitalization 2011 to 2012. HCUP Projections Report \# 2012-01.

Sun, X., Wang, H., Zhang, Y., Chen, K., Davis, B., and Feng, H. (2011). Mouse relapse model of Clostridium difficile infection. Infect. Immun. 79, 2856-2864. doi: 10.1128/IAI.01336-10

Sunenshine, R. H., and McDonald, L. C. (2006). Clostridium difficile-associated disease: new challenges from an established pathogen. Cleve. Clin. J. Med. 73, 187-197. doi: 10.3949/ccjm.73.2.187

Theriot, C. M., Koenigsknecht, M. J., Carlson, P. E., Hatton, G. E., Nelson, A. M., Li, B., et al. (2014). Antibiotic-induced shifts in the mouse gut microbiome and metabolome increase susceptibility to Clostridium difficile infection. Nat. Commun. 5:3114. doi: 10.1038/ncomms4114

Vesper, B. J., Jawdi, A., Altman, K. W., Haines, G. K. III, Tao, L., Radosevich, J. A., et al. (2009). The effect of proton pump inhibitors on the human microbiota. Curr. Drug Metab. 10, 84-89. doi: 10.2174/138920009787048392

von Eichel-Streiber, C., Zec-Pirnat, I., Grabnar, M., and Rupnik, M. (1999). A nonsense mutation abrogates production of a functional enterotoxin $\mathrm{A}$ in Clostridium difficile toxinotype VIII strains of serogroups $\mathrm{F}$ and X. FEMS Microbiol. Lett. 178, 163-168.

Voth, D. E., and Ballard, J. D. (2005). Clostridium difficile toxins: mechanism of action and role in disease. Clin. Microbiol. Rev. 18, 247-263. doi: 10.1128/CMR.18.2.247-263.2005 
Weese, J. S. (2010). Clostridium difficile in food-innocent bystander or serious threat? Clin. Microbiol. Infect. 16, 3-10. doi: 10.1111/j.1469-0691.2009. 03108.x

Weingarden, A. R., Chen, C., Bobr, A., Yao, D., Lu, Y., Nelson, V. M., et al. (2014). Microbiota transplantation restores normal fecal bile acid composition in recurrent Clostridium difficile infection. Am. J. Physiol. Gastrointest. Liver Physiol. 306, G310-G319. doi: 10.1152/ajpgi.0028 2.2013

Wilkins, T. D., and Lyerly, D. M. (2003). Clostridium difficile testing: after 20 years, still challenging. J. Clin. Microbiol. 41, 531-534. doi: 10.1128/JCM.41.2.531-534.2003
Conflict of Interest Statement: The authors declare that the research was conducted in the absence of any commercial or financial relationships that could be construed as a potential conflict of interest.

Copyright (C) 2017 Mooyottu, Flock, Upadhyay, Upadhyaya, Maas and Venkitanarayanan. This is an open-access article distributed under the terms of the Creative Commons Attribution License (CC BY). The use, distribution or reproduction in other forums is permitted, provided the original author(s) or licensor are credited and that the original publication in this journal is cited, in accordance with accepted academic practice. No use, distribution or reproduction is permitted which does not comply with these terms. 